\title{
Object Classification Based on Radon Transform
}

\author{
Jing Ma, Baolong Guo \\ Institute of Intelligent Control and Image Engineering, Xidian University, Xi'an 710071, China \\ mjy_9527@126.com
}

Keywords: Automatic Target Recognition (ATR), Radon Transform, Support Vector Machine (SVM) Classifier.

Abstract: With the development of science and technology, the speed of taking and transmitting images has greatly improved, so that the target recognition technology based on remote sensing image becomes a reality. According to the deficiencies of the previous recognition methods, a method of automatic threshold is proposed for getting more accurate features about the positioned object, then the feature extraction by SVM classifier for classification and recognition. Experiments demonstrate the effectiveness of the current recognition system. The system provides a good performance in aircraft recognition and classification, at the same time it also offers better robustness.

\section{Introduction}

At present, in target automatic recognition we usually use multispectral image, satellite remote sensing image to recognize target, mainly for typical targets (such as bridges, ports, airports, etc.). In aspect of targets, mostly major in some algorithms researches [1-5], from the requirements of real time, accuracy and reliability have a certain distance. For the visible light image, difficulties in recognition lies in that the target has no obvious common gray feature and the background is very complex. The automatic binaryzation algorithm that can identify aircraft is a frequently-used method and several common methods such as invariants [6], Fourier descriptors [7] or geometric invariants are used as features for recognition and classification.

In the article, we take the satellite remote sensing image as an example. After locating of suspected target in the picture, transform the gray image into binary image by iterative threshold binarization, restore the fracture place in the original image. And then extract characteristics from the above results, put those characteristic into a filter. It will be discarded, if the object is not the target. Otherwise we will make use of SVM [8] classifier to get the result.

\section{Image Preprocessing}

Satellite remote sensing image exist lots of noise, so it needs to be processed before operation. Gray image and color image can reflect the distribution and characteristics of the whole image of the global and local color and brightness level. This paper uses the next type of remote sensing image weighted average gray.

$$
\text { gray }=0.399 \times \mathrm{r}+0.587 \times \mathrm{g}+0.114 \times \mathrm{b} \text {. }
$$


In the above formula, $r, g, b$ represent the remote sensing images of red, green, blue color channel component, gray represents the value of pixel after gray processing. After the end of gray, the next necessary step is de-noising. At first, adaptive median filtering is used for reducing the noise. And then to reduce the band noise through frequency filter based on Fourier trans-form is adopted.

In order to overcome the negative effects of the multiple factors such as the complexity of the target, the environmental changes, scale differences, data quality and so on. Firstly, a method based on Harris corner detection is used to position aircraft. The location results are shown in Fig.1, the blue box indicates the aircraft area.

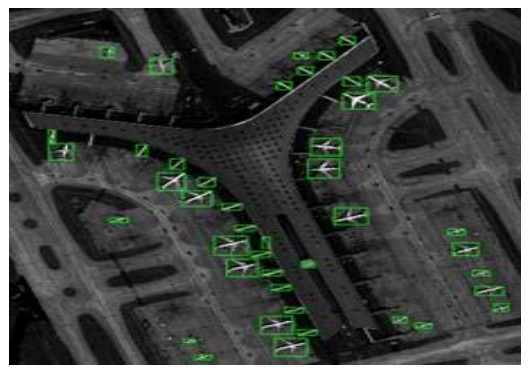

Fig. 1. Aircraft detection result

\section{Feature Extraction}

For the satellite remote sensing image which can't get the details of the aircraft, in order to classify the aircraft, the original feature extraction method can't describe the characteristics of the aircraft very well. In this paper we take the Radon change and the invariant moments as the aircraft characteristics.

Radon Transform. The original images often need a conversion to other space in order to carry on the processing effectively and quickly. And make use of the special nature of those spaces to carry out a certain processing, in the end convert the image to the previous image space to get the desired results.

Radon transform can be defined in any dimensional space, for the image, consider the transformation form of two-dimensional Euclidean space.

$$
R(\theta, \rho)=\iint f(x, y) \delta(s-x \cos \theta-y \sin \theta) d x d y
$$

In the equation $f(x, y)$ is the gray value of a pixel in the image, characteristic function $\delta$ is Dirac function, $\mathrm{s}$ represents the distance from the straight line in the $(\mathrm{x}, \mathrm{y})$ plane to the origin, $\theta$ represents the angle between the vertical line of the origin to the straight line and the $\mathrm{x}$ axis. The radon transform of $f(x, y)$ can be expressed as Fig. 2.

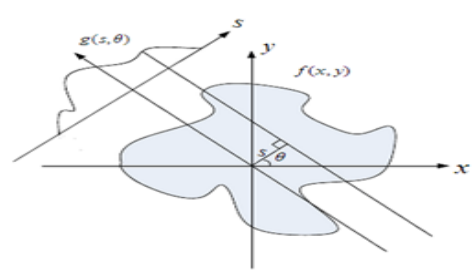

Fig. 2. Radon transform diagram

Because the aircraft models are fixed that most of the aircraft fuselage is longer and the wing is prominent, after integrating the aircraft in all directions, as you can see in Fig. 3, the integral value along the fuselage and wing direction is relatively large. Different aircraft peak position and height 
are different, the same aircraft peak position and height are similar. So the radon values as shown in Fig. 5 can be used as the feature to classify the aircraft.

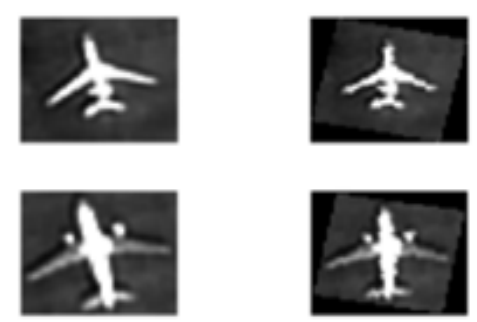

Fig. 3. Radon angular adjustment

Different aircraft peak position and height are different, the same aircraft peak position and height are similar. In this paper we choose the following four points to carry out the Radon transform. The radon values as shown in Fig.4 can be used as the feature to classify the aircraft.
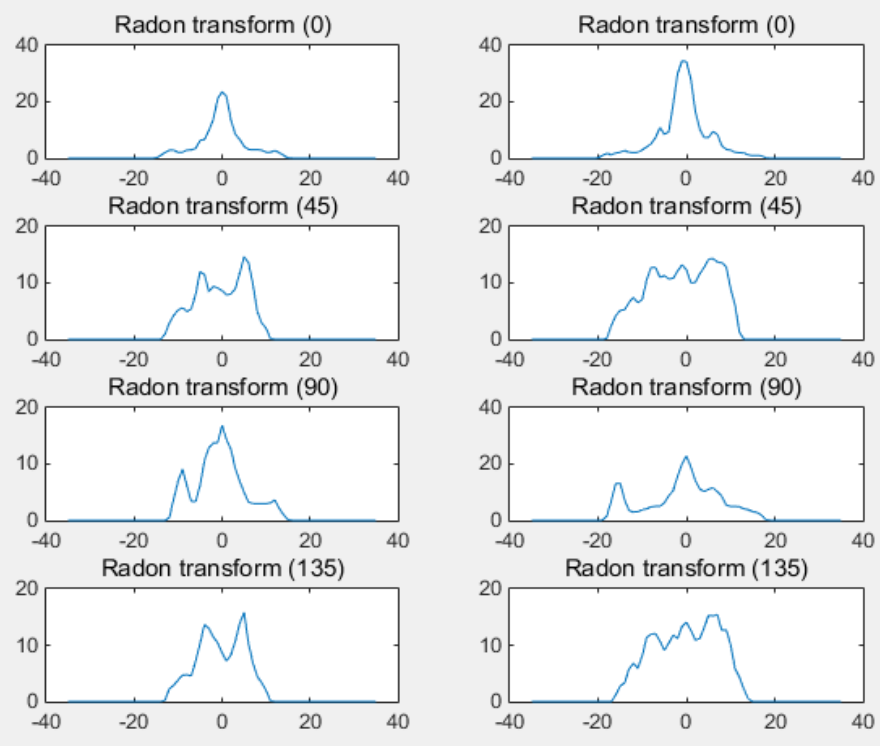

Fig. 4. Radon transform in different directions

\section{SVM classifier}

For target detection, there is no better method that can detect all of the target accurately in remote sensing image. Before the classification, we first to extract the features from all suspected targets, through the classifier to filter out the target of false detection, and then use the SVM for classification. For aircraft identification, the classification algorithm used in this paper is as follows: 


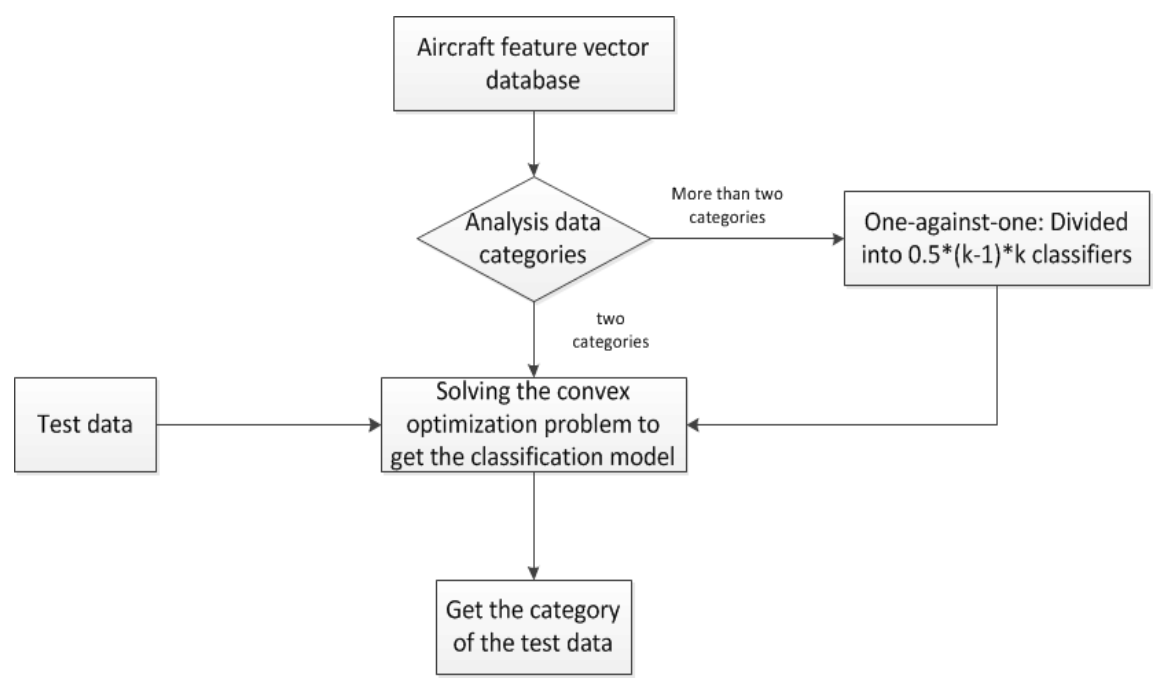

Fig. 5. The flowchart of SVM classifier in this paper

In this paper, we choose 200 aircraft as the original data, randomly selected 30 of them as a test set, and the rest as a training set. In order to make the result more visualized, we did three groups of tests. The first method is proposed in this paper, which adopts the Radon transform of different directions and $\mathrm{Hu}$ invariant moments as features, and the SVM classifier is used for classification. As can be seen from the table, the average recognition rate of the first method is 93.2 percent. The second experiment is using the $\mathrm{Hu}$ invariant moments and simple geometric shape information as the characteristics of the aircraft, and then we use the same SVM classifier as the first experiment. From the Table, we can see that the average recognition rate is 89.5 percent. In the last experiment, we use the same method as the first experiment to extract features, and then use random forest classification. Its recognition rate is 86 percent, which is lower than other two methods.

Table 1 . The result of classify

\begin{tabular}{|c|c|c|c|}
\hline $\begin{array}{c}\text { Classification } \\
\text { Method }\end{array}$ & $\begin{array}{c}\text { SVM classication based } \\
\text { on Radon transform }\end{array}$ & $\begin{array}{c}\text { SVM classification } \\
\text { based on Hu's invariants } \\
\text { and Simple geometric } \\
\text { features }\end{array}$ & $\begin{array}{c}\text { Random forest } \\
\text { classification based on } \\
\text { Radon transform }\end{array}$ \\
\hline 1 & $92.0 \%$ & $90.2 \%$ & $86.3 \%$ \\
\hline 2 & $95.3 \%$ & $86.0 \%$ & $83.0 \%$ \\
\hline 3 & $88.6 \%$ & $88.7 \%$ & $85.6 \%$ \\
\hline 4 & $96.8 \%$ & $93.0 \%$ & $88.9 \%$ \\
\hline Average & $93.2 \%$ & $89.5 \%$ & $86.0 \%$ \\
\hline
\end{tabular}

Through the above analysis, we can see that the combination of Radon transform features and SVM classifier is better than the other two methods. Fig.6 shows the local classification of the results, 2 represent the engine front of the aircraft.

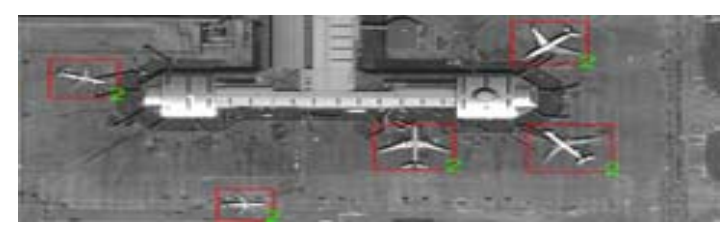

Fig. 6. Classification results in graphs 


\section{Summary}

In this paper, firstly, we study a good method to get the specimen, which is based on image preprocessing and then, on this base, we get the main characteristic after Radon transform. At last, the SVM classifier is used for classification, and the result is obvious.

\section{Acknowledgements}

This work was financially supported by the National Natural Science Foundation of China under Grants No. 61571346.

\section{References}

[1] Xufeng Zhu., Caiwen Ma.: The Study of Combined Invariants Optimization Method on Aircraft Recognition, Symposium on Photon-ics and Optoelectronics. IEEE Press, 1-4(2011)

[2] Wei Li., Shiming Xiang., Haibo Wang., Chunhong Pan.: Robust Airplane Detection in Satellite Images, International Conference on Image Processing. IEEE Press, 2821-2824(2011)

[3] J.-W Hsieh., J.-M Chen., C.-H Chuang., et al.: Aircraft Type Recognition in Satellite Images.

[4] IEE Proceedings on Vision, Image and Signal Processing, 152(3): 307-315(2005)

[5] Ge Liu., Xian Sun., Kun Fu., et al.: Aircraft Recognition in High-resolution Satellite Images Using Coarse-to-fine Shape Prior. IEEE Geoscience and Remote Sensing Letters, 10(3): 573-577(2013)

[6] Hu.: Visual Pattern Recognition by Moment Invariants. IRE Transactions on Information Theory, 2(8): 179-187(1962)

[7] GE Qiaode Jeffrey.: Fourier Descriptors with Different Shape Sig-natures: A Comparative Study for Shape Based Retrieval of Kinematic Constraints. Chinese Journal of Mechanical Engineering, 05:723-730(2011)

[8] M., Deng Y.: SVM Classifier - a comprehensive java interface for support vector machine classification of microarray data. Bmc Bioinformatics, 7(Suppl 4):1-7(2006) 